\title{
Voxel-Based Morphometry and fMRI Revealed Differences in Brain Gray Matter in Breastfed and Milk Formula-Fed Children
}

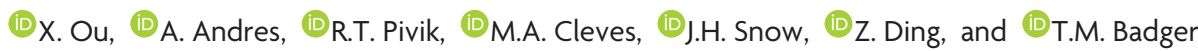

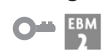

\begin{abstract}
BACKGROUND AND PURPOSE: Infant diets may have significant impact on brain development in children. The aim of this study was to evaluate brain gray matter structure and function in 8-year-old children who were predominantly breastfed or fed cow's milk formula as infants.

MATERIALS AND METHODS: Forty-two healthy children (breastfed: $n=22,10$ boys and 12 girls; cow's milk formula: $n=20,10$ boys and 10 girls) were studied by using structural MR imaging (3D T1-weighted imaging) and blood oxygen level-dependent fMRI (while performing tasks involving visual perception and language functions). They were also administered standardized tests evaluating intelligence (Reynolds Intellectual Assessment Scales) and language skills (Clinical Evaluation of Language Fundamentals).

RESULTS: Total brain gray matter volume did not differ between the breastfed and cow's milk formula groups. However, breastfed children had significantly higher $(P<.05$, corrected) regional gray matter volume measured by voxel-based morphometry in the left inferior temporal lobe and left superior parietal lobe compared with cow's milk formula-fed children. Breastfed children showed significantly more brain activation in the right frontal and left/right temporal lobes on fMRI when processing the perception task and in the left temporal/occipital lobe when processing the visual language task than cow's milk formula-fed children. The imaging findings were associated with significantly better performance for breastfed than cow's milk formula-fed children on both tasks.
\end{abstract}

CONCLUSIONS: Our findings indicated greater regional gray matter development and better regional gray matter function in breastfed than cow's milk formula-fed children at 8 years of age and suggested that infant diets may have long-term influences on brain development in children.

ABBREVIATIONS: BF = breastfed; $C$ ELF-4 = Clinical Evaluation of Language Fundamentals, Fourth Edition; $\mathrm{IQ}=$ intelligent quotient; $M$ F $=$ cow's milk formula-fed; RIAS = Reynolds Intellectual Assessment Scales; VBM = voxel-based morphometry

$\mathbf{T}$ he World Health Organization recommends exclusive breastfeeding, which has been associated with better school performance and neurocognitive development in children by multiple studies. ${ }^{1-5}$ When breastfeeding is not possible or not chosen, cow's milk formula has been frequently the formula of choice. Milk formula is made with bovine milk protein, and while the

\section{Received July 10, 2015; accepted after revision September 10}

From the Arkansas Children's Nutrition Center (X.O., A.A., R.T.P., M.A.C., T.M.B.) and Departments of Pediatrics (X.O., A.A., R.T.P., M.A.C., J.H.S., T.M.B.) and Radiology (X.O.), University of Arkansas for Medical Sciences, Little Rock, Arkansas; and Vanderbilt University Institute of Imaging Sciences (Z.D.), Nashville, Tennessee.

This work was funded by US Department of Agriculture/Agricultural Research Service 6251-51000-005-02.

Please address correspondence to Xiawei Ou, PhD, One Children's Way, Slot 105, Little Rock, AR 72202; e-mail: ouxiawei@uams.edu

-- Indicates open access to non-subscribers at www.ajnr.org

EBM Evidence-Based Medicine Level 2.

http://dx.doi.org/10.3174/ajnr.A4593 amino acid composition is similar to that of human breast milk, there are differences in the overall composition of milk formula and human breast milk. It is possible that these differences can impact the developing brain and therefore contribute to the observed long-term benefits on neurodevelopment associated with breastfeeding. In fact, studies are beginning to appear showing an association between breastfeeding and better development in brain structures, such as increased white matter volume, greater cortical thickness, and increased microstructural integrity and myelin water fraction in white matter. ${ }^{6-9}$

Rapid advances of neuroimaging methods ${ }^{10}$ have made possible more comprehensive evaluation of brain development in children. For example, regional gray matter volume measured by MR imaging voxel-based morphometry (VBM) provides a wholebrain region-specific evaluation of the deep gray matter and brain cortex. ${ }^{11}$ Gray matter consists mostly of neuronal cell bodies that generate and process nerve signals underlying brain function. $\mathrm{Nu}$ merous VBM studies have revealed abnormal gray matter in dif- 
ferent brain regions in children with a variety of neurodevelopmental or psychological disorders. ${ }^{12-14} \mathrm{VBM}$ analysis of infant diet effects has not been previously reported, to our knowledge. However, other dietary factors, such as breakfast composition, have shown effects on regional gray matter volume in children. ${ }^{15}$

Dietary effects on brain gray matter revealed by structural MR imaging may be complemented by measures of brain functioning obtained by using fMRI. Currently, there is a lack of literature on the use of fMRI to study differences in brain functioning associated with infant diets. For example, although breastfeeding has been associated with better receptive and expressive language development in children ${ }^{16}$ and longer breastfeeding duration was associated with higher receptive language scores at 3 years of age and with higher verbal intelligence quotient (IQ) at 7 years of age, ${ }^{17}$ it is not known whether fMRI procedures would reveal diet-specific effects during brain processing on language tasks in children fed different diets as infants.

The aim of this study was to evaluate brain gray matter structure and function in 8-year-old healthy children who were fed predominantly breast milk (BF) or cow's milk formula (MF) during the first year of life. We hypothesized that breastfeeding benefits brain gray matter development and functioning in children. To test this hypothesis, we compared VBM measures of regional gray matter volume and fMRI measures of brain activation when performing visual perception and language tasks between BF and MF children. Measures of fMRI task performance and overall IQ and language skills were also compared between groups.

\section{MATERIALS AND METHODS Participants}

Healthy 7.5-8.5-year-old children who had parental report of predominant breast milk or cow's milk-based formula feeding during infancy were recruited for this study. All procedures were approved by the University of Arkansas for Medical Sciences review board. Assents and consents were obtained from the participants and their parents. BF infants were all exclusively breastfed for at least 8 months (average, 12.6 months). MF infants were fed the same type of cow's milk-based formula after birth for at least 8 months. Infants were introduced to complementary foods on average around 5 months of age. All participants were righthanded and born full-term with birth weight between the fifth and 95th percentiles. None had a medical history of psychologi$\mathrm{cal} / \mathrm{psychiatric} \mathrm{diagnoses} \mathrm{or} \mathrm{neurologic} \mathrm{impairment} \mathrm{or} \mathrm{injury.} \mathrm{In}$ addition, none of their mothers reported alcohol, tobacco, drug, or psychotropic medications use during pregnancy. All participants underwent an MR imaging examination including a structural scan and an fMRI study. After we excluded those who did not complete the MR imaging scan or had excessive motion and apparent imaging artifacts, valid structural imaging data were obtained for 42 children (BF: $n=22,10$ boys, 12 girls; MF: $n=20$, 10 boys, 10 girls), and all except 1 MF girl had valid fMRI data. Participants were administered the Reynolds Intellectual Assessment Scales (RIAS) test, which measures verbal, nonverbal, and composite IQ, and the Clinical Evaluation of Language Fundamentals (CELF-4) test, which measures receptive, expressive, and overall language abilities. The RIAS and CELF-4 tests were supervised by a licensed pediatric psychological examiner. In addition,
Table 1: Demographic information, family background, and test scores of the study participants

\begin{tabular}{|c|c|c|c|}
\hline & $\mathrm{BF}$ & MF & $P$ Value \\
\hline \multicolumn{4}{|l|}{ Demographics } \\
\hline No. (boys/girls) for VBM & $22(10 / 12)$ & $20(10 / 10)$ & \\
\hline Birth weight (kg) & $3.5 \pm 0.4$ & $3.4 \pm 0.5$ & .47 \\
\hline Weight (kg) & $27.8 \pm 6.3$ & $27.5 \pm 5.5$ & .98 \\
\hline Height (cm) & $128 \pm 5$ & $129 \pm 4$ & .25 \\
\hline BMI & $17.0 \pm 3.4$ & $16.5 \pm 2.6$ & .62 \\
\hline Age at MRI (yr) & $7.98 \pm 0.29$ & $7.95 \pm 0.25$ & .71 \\
\hline \multicolumn{4}{|l|}{ Family background } \\
\hline Mother's education ${ }^{\mathrm{a}}(\%)$ & $35.7 / 42.9 / 21.4$ & $8.3 / 50 / 41.7$ & .24 \\
\hline Father's education ${ }^{a}(\%)$ & $35.7 / 50 / 14.3$ & $33.3 / 58.3 / 8.3$ & .99 \\
\hline Household income ${ }^{\mathrm{b}}(\%)$ & 21.4/57.1/21.4 & 0/41.7/58.3 & .08 \\
\hline \multicolumn{4}{|l|}{ Test scores } \\
\hline Verbal IQ & $110 \pm 16$ & $107 \pm 17$ & .50 \\
\hline Nonverbal IQ & $111 \pm 13$ & $114 \pm 16$ & .73 \\
\hline Composite IQ & $111 \pm 13$ & $110 \pm 15$ & .68 \\
\hline CELF-4 (receptive) & $104 \pm 11$ & $100 \pm 15$ & .45 \\
\hline CELF-4 (expressive) & $108 \pm 10$ & $104 \pm 12$ & .33 \\
\hline CELF-4 (overall) & $104 \pm 12$ & $100 \pm 15$ & .41 \\
\hline
\end{tabular}

Note:-BMI indicates body mass index.

${ }^{a}$ High school or partial college/college graduate/graduate school. b $<\$ 50,000 / \$ 50,000-\$ 70,000 />\$ 70,000$.

we were able to obtain family background information, including parents' education and income, for 26 subjects (12 MF and $14 \mathrm{BF}$ children). The demographic information, family background, and test scores for all subjects are listed in Table 1.

\section{MR Imaging Protocol}

All MR imaging scans were performed on a 1.5T Achieva scanner (Philips Healthcare, Best, the Netherlands) with a $60-\mathrm{cm}$ bore size, 33-mT/m gradient amplitude, and $100-\mathrm{mT} / \mathrm{m} / \mathrm{ms}$ maximum slew rate at our institution. The built-in body coil was used as a transmitter, and a standard 8-channel sensitivity encoding head coil was used as a receiver. Imaging sequences included a sagittal T1-weighted 3D turbo field echo sequence for structural MR imaging with the following parameters: TR, $7.3 \mathrm{~ms}$; TE, $3.4 \mathrm{~ms}$; flip angle, $8^{\circ}$; acquisition voxel size, $1 \times 1 \times 1 \mathrm{~mm}$; matrix size, $256 \times$ $232 \times 150 ; 2$ averages; scan time, $\sim 7$ minutes; and an axial singleshot gradient-echo-planar imaging sequence for functional MR imaging with the following parameters: TR, $3000 \mathrm{~ms}$; TE, $50 \mathrm{~ms}$; flip angle, $90^{\circ}$; acquisition voxel size, $2.4 \times 2.4 \times 5 \mathrm{~mm}$; matrix size, $92 \times 92 \times 20$; 208 dynamic scans; 2 dummy scans; scan time, $\sim 10$ minutes.

\section{Structural MR Imaging Data Processing}

The raw T1-weighted 3D images were exported to a workstation with Matlab software (MathWorks, Natick, Massachusetts) for VBM analysis. The VBM8 toolbox for statistical parametric mapping (SPM8; http://www.fil.ion.ucl.ac.uk/spm/software/spm8) was used. A pediatric T1 template ( $\sim 8$ years of age) and customized tissue probability maps were created on the basis of National Institutes of Health data by the Template-O-Matic toolbox (https://irc.cchmc.org/software/tom/agreement.php), ${ }^{18}$ and the T1 images were then segmented into gray matter, white matter, and CSF by VBM8 by using default parameters. Nonlinear modulated normalized gray matter images were generated that enable analysis of relative differences in regional gray matter volume, corrected for individual brain size. The images were displayed and 
reviewed to ensure that segmentation and normalization procedures worked properly and were then smoothed by using a Gaussian kernel of full width at half maximum, $8 \mathrm{~mm}$ in each direction. The smoothed gray matter images were used for the subsequent voxelwise statistical analysis between the BF and MF groups. Although there were no significant sex or age differences between groups, sex and age were included as covariates in the VBM analysis because the brain is still developing at 8 years of age and there are possible sex differences in the human brain. ${ }^{19}$

\section{fMRI Protocol}

All participants were trained before the fMRI study to perform the perception and language tasks. The tasks were adopted from the literature ${ }^{20}$ with modifications regarding timing and design and incorporated into an fMRI paradigm designed on E-Prime software (Psychology Software Tools, Pittsburgh, Pennsylvania). For the perception task, the participants were requested to determine whether 2 symbols on the screen (ie, $\wedge$ ) matched those presented on the previous screen (eg, $\backslash \backslash, / /, \backslash /$ ). For the language task, participants were requested to determine whether 2 words presented on 1 screen after another rhymed. A block design consisting of 8 perception blocks and 8 language blocks alternating with each other was used for the fMRI. Each block included 9 trials, and each trial included presentation of symbols (perception block) or words (rhyming block) followed by a 2000-ms interval during which the participants were to make responses by pressing either the "yes" or "no" key on a touchpad of an Eloquence fMRI system (Invivo, Gainesville, Florida).

\section{fMRI Data Processing}

Gradient-echo EPI acquired during fMRI examinations was exported to a workstation with BrainVoyager software (Brain Innovation, Maastricht, the Netherlands) for fMRI data processing. Cubic spline interpolation was used for slice scan time correction. Images from the first dynamics were used as a reference, and translation and rotation of images in subsequent dynamics were plotted in all directions to illustrate motion. Trilinear estimation and interpolation were used for 3D-motion correction. An 8-mm full width at half maximum Gaussian filter was used for spatial smoothing. Linear trend removal and a high-pass filter with 3 cycles/points were used for temporal filtering. The T1-weighted $3 \mathrm{D}$ images were also exported to BrainVoyager to create an anatomic image series, and the processed fMRI was coregistered to the anatomic images automatically. The 3D dataset with anatomic images and fMRI information was then transformed to the Talairach atlas to create a 3D-aligned time course dataset. A stimulation protocol was then created in BrainVoyager to represent the block design (with hemodynamic response function refinement) used in the fMRI scans. General linear model analysis was performed to calculate activation maps for the 3D-aligned time course dataset for each subject. The average activation maps for each diet group were calculated by a multistudy multisubject general linear model. The total activation cluster size in each brain lobe that exhibited activation at a statistical threshold of $P<.001$ corrected was calculated for each subject and compared between groups. In addition, task performance parameters, such as the percentage of responses and the percentage of correct answers

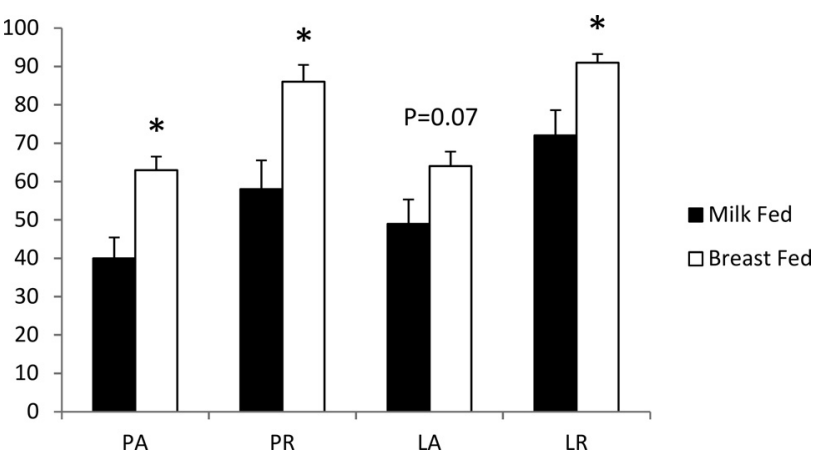

FIG 1. BF children had better performance for $\mathrm{fMRI}$ perception and language tasks. PA indicates percentage of correct answer for the perception task; PR, percentage of response for the perception task; LA, percentage of correct answer for the language task; LR, percentage of response for the language task. The asterisk indicates $P<.05$.

made within the allocated time interval in each trial, were also compared between groups.

\section{Statistical Analysis}

For comparisons of demographic parameters, IQ and language test scores, total gray matter volume, and fMRI task performance parameters among groups, Wilcoxon rank sum tests were used for significant differences $(P<.05)$. Comparison of other non-numeric family background parameters were made by using the Fisher exact test. For the VBM comparisons of regional gray matter volume among groups, $P<.001$ uncorrected with a cluster size threshold of 30 voxels was used to illustrate overall differences, and $P<.05$ (family-wise error-corrected cluster level) was used to determine regions with significantly different regional gray matter volumes. Sex and age were included as covariates for all analyses. For the calculation and illustration of average fMRI activation maps in each group, the statistical threshold was set at $P<.001$ after Bonferroni correction for multiple comparisons and the cluster size threshold was set at 30 voxels. For the statistical comparison of fMRI activation cluster size between groups, $P<.001$ after Bonferroni correction (for the activation maps) was used to calculate total cluster size in each brain lobe in each subject, and Wilcoxon rank sum tests were used to determine significant differences $(P<.05)$ between groups.

\section{RESULTS}

The weight, height, body mass index, and age at MR imaging were not different between the BF and MF children (Table 1). The family background parameters were also not different between groups. The RIAS IQ and CELF-4 scores did not differ between BF and MF. However, BF children performed better on both fMRI tasks, as indicated by the percentage of responses made within the designated time interval and the percentage of correct answers (Fig 1) in which BF and MF children differed significantly on 3 of the 4 measures.

Total gray matter volume $\left(763 \pm 65 \mathrm{~cm}^{3}\right.$ and $761 \pm 78 \mathrm{~cm}^{3}$ for the $\mathrm{BF}$ and MF children, respectively) did not differ between groups. However, BF children had higher regional gray matter volume than MF children in multiple brain regions (Fig 2). Specifically, at a statistical threshold of $P<.001$ (uncorrected) with a cluster size of $\geq 30$ voxels, regional gray matter volume was 
greater for BF than MF children in the left and right parietal and left temporal lobes (Fig $2 A$ ), while there were no regions with lower gray matter volume (Fig $2 B$ ). At a statistical threshold of $P<.05$ (family-wise error-corrected), 1 region in the left supe-
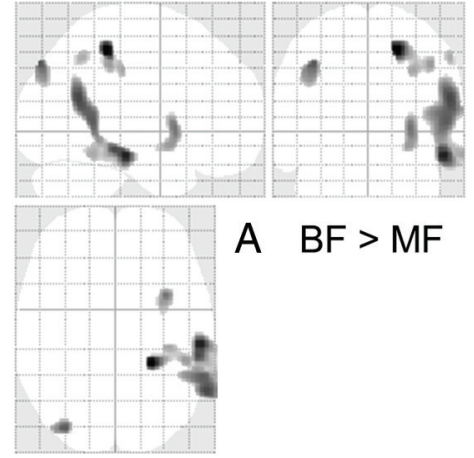

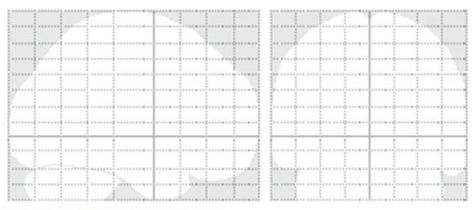

B $\quad \mathrm{MF}>\mathrm{BF}$

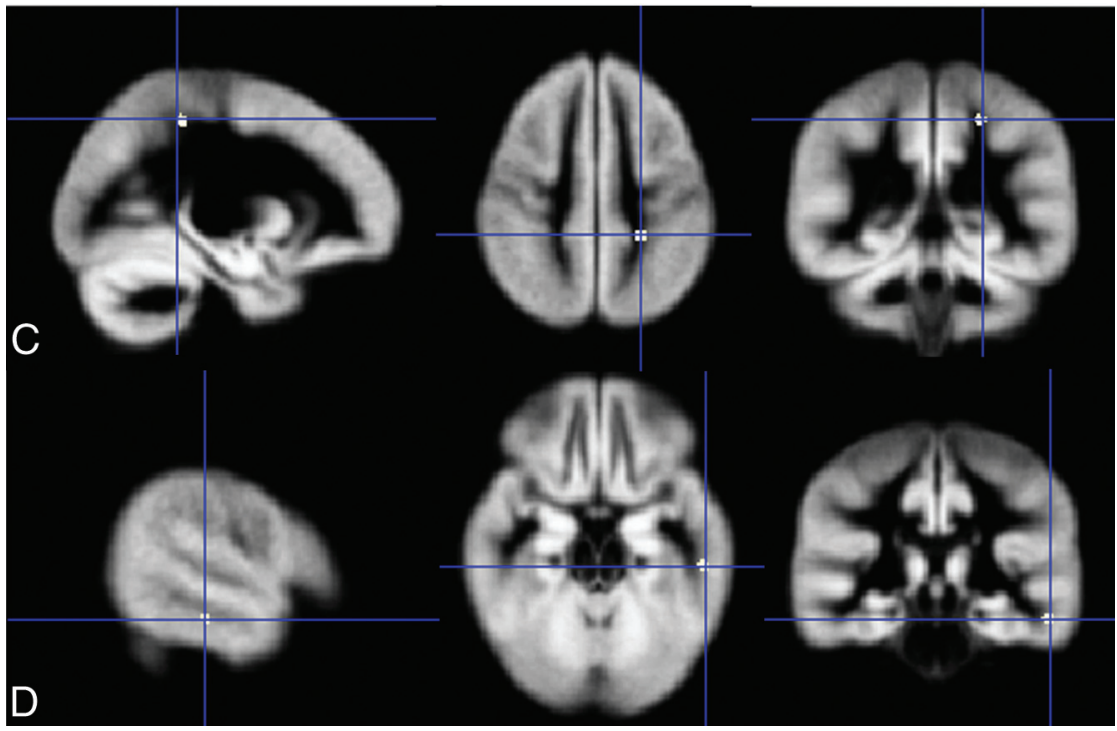

FIG 2. Comparison of regional gray matter volume measures by VBM. The results are shown in a 3 -plane projection view in $A$ and $B$, in which the statistical threshold was set at $P<.001$ uncorrected, with a cluster size of $\geq 30$ voxels. $A$, Multiple regions had higher gray matter volumes in $B F$ than MF children. $B$, No region had higher gray matter volume in MF than BF children. Regions with significantly higher $(P<.05$, corrected) gray matter volume in BF than MF children were overlaid on the customized gray matter template for 8-year-old children (1 region in the left superior parietal lobe, $C$, and 1 region in the left inferior temporal lobe, $D)$. All images are displayed in radiologic convention (left/right flipped). rior parietal lobe (Fig $2 C$ ) and 1 region in the left inferior temporal lobe (Fig $2 D$ ) had significantly higher gray matter volume in BF than MF children, and no region had lower gray matter volume.

Average fMRI activation maps for the perception task (Fig 3; $P<.001$, Bonferroni-corrected cluster size of $\geq 30$ voxels) showed widespread activation in the left and right middle temporal gyri, superior parietal gyrus/precuneus, and right superior/middle frontal gyri for $\mathrm{BF}$ children, while MF children had fewer activation regions, which mainly involved the right middle temporal gyrus and superior parietal gyrus/precuneus. Average fMRI activation maps for the language task (Fig 4, $P<.001$, Bonferroni-corrected cluster size of $\geq 30$ voxels) showed that BF children had widespread activation in the left and right lingual gyri and cuneus and left fusiform and the left middle/inferior frontal gyri, while MF children had similar overall activation except for less left temporal lobe activation. The total activation cluster size $(P<.001$, Bonferroni-corrected) calculated for individual subjects in each of the involved brain areas (Table 2) showed significantly more activation for BF children in the right frontal and left and right temporal lobes for the perception task and in the left temporal/ occipital lobes for the language task (all, $P<.05)$.

\section{DISCUSSION}

The total brain gray matter volume did not differ between BF and MF children in our study. This is consistent with a previous report that did not observe a significant correlation between breast

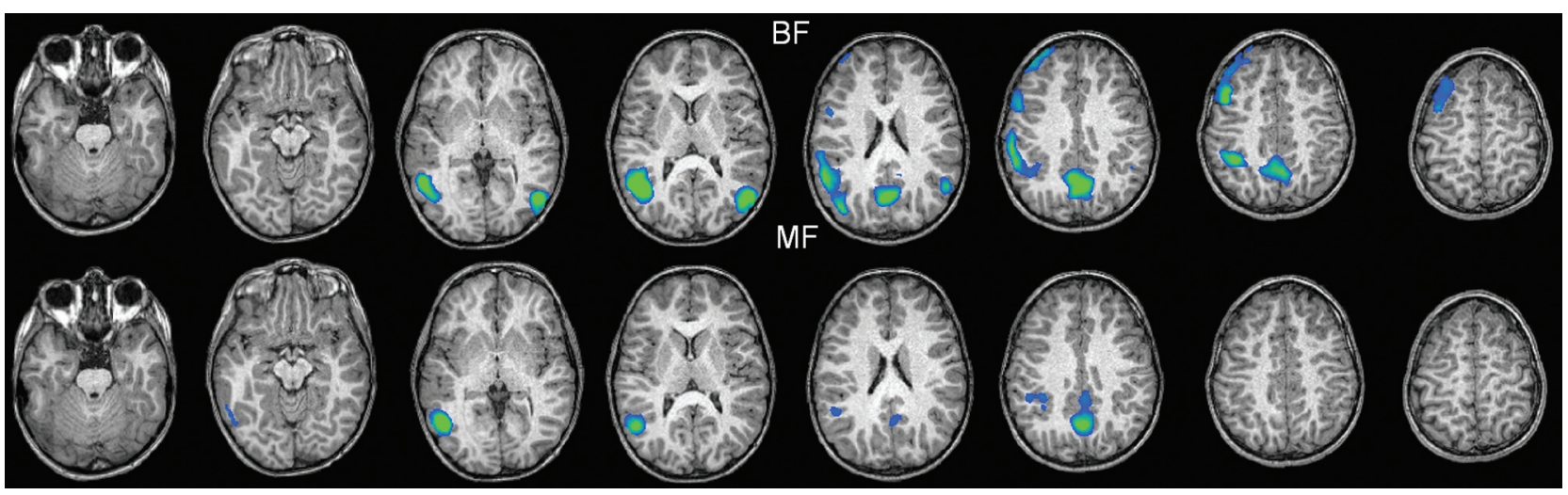

FIG 3. Average activation maps for the fMRI perception task for BF (top row) and MF (bottom row) children. Activated regions are highlighted in blue/green. The threshold was set at $P<.001$ after Bonferroni correction and a cluster size of $\geq 30$ voxels. All images are displayed in radiologic convention (left/right flipped). BF children had more extensive activation in the right frontal and left/right temporal lobes. 


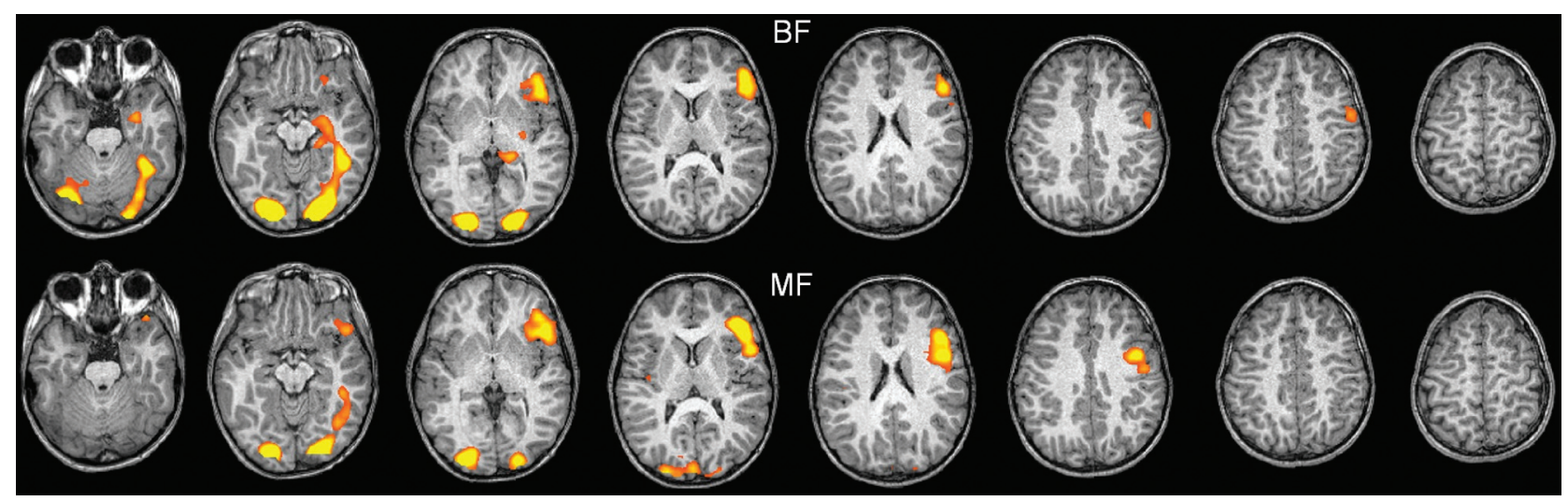

FIG 4. Average activation maps for the fMRI language task for BF (top row) and MF (bottom row) children. Activated regions are highlighted in yellow/orange. The threshold was set at $P<.001$ after Bonferroni correction and a cluster size of $\geq 30$ voxels. All images are displayed in radiologic convention (left/right flipped). BF children had more extensive activation in the left temporal lobe.

Table 2: Comparison of the fMRI-activation total cluster size (No. of voxels) ${ }^{a}$

\begin{tabular}{lccc}
\hline & $\begin{array}{c}\text { BF Mean } \\
\text { (SE) }\end{array}$ & $\begin{array}{c}\text { MF Mean } \\
\text { (SE) }\end{array}$ & $\boldsymbol{P}$ Value \\
\hline Perception fMRI & & & \\
$\quad$ Right frontal & $1280(814)$ & $6(6)$ & $.002^{\mathrm{b}}$ \\
$\quad$ Parietal & $1263(488)$ & $723(546)$ & .445 \\
$\quad$ Left temporal & $1394(680)$ & $112(86)$ & $.011^{\mathrm{b}}$ \\
$\quad$ Right temporal & $2263(1364)$ & $33(22)$ & $.041^{\mathrm{b}}$ \\
Language fMRI & & & \\
$\quad$ Left frontal & $1125(616)$ & $261(113)$ & .513 \\
$\quad$ Left temporal/occipital & $2632(650)$ & $613(213)$ & $.04^{\mathrm{b}}$ \\
$\quad$ Right temporal/occipital & $1894(609)$ & $909(369)$ & .251 \\
\hline
\end{tabular}

Note:-SE indicates standard error.

${ }^{a} B F$ children had greater activation than MF children for both fMRI tasks. ${ }^{\mathrm{b}} \mathrm{P}<.05$.

milk intake and total gray matter volume in ex-preterm children. ${ }^{6}$ However, these measures of total volume provide a gross assessment of brain structure and may not be sensitive enough to detect subtle and/or regional differences. On the other hand, regional gray matter volume analysis by VBM used in the present study can detect regional differences in the distribution of gray matter, in addition to providing whole-brain analyses. Our results revealed multiple brain regions with significantly higher gray matter volume in BF than in MF children. These findings are consistent with previous reports of better regional gray matter development associated with breastfeeding compared with formula feeding. ${ }^{7,21}$ Our VBM analysis observed significant regional gray matter differences in BF-versus-MF children in cortical but not deep gray matter. These results differ from those of Herba et al, ${ }^{21}$ who showed a larger gangliothalamic ovoid diameter in BF-versus-formula-fed infants as quantified by using sonography. These apparent discordant results may be influenced by the brain maturation pattern of deep gray matter, which develops earlier than cortical gray mat$\operatorname{ter}^{22}$; thus, diet effects on gray matter may be more prominent in deep regions during infancy, but in cortical regions during later childhood. Our VBM analysis identified higher regional gray matter volume in the parietal lobe of BF-versus-MF children, in agreement with Kafouri et al, ${ }^{7}$ who reported a positive association between the duration of exclusive breastfeeding and cortical thickness of superior and inferior parietal lobules. In addition, our study also revealed significant regional gray matter volume differences between BF and MF children in the left inferior temporal lobe, indicating that voxelwise whole-brain analysis may be more sensitive than the ROI cortical thickness measure. Finally, the regional gray matter volume comparison by VBM was based on modulated gray matter attenuation, while the total gray matter volume was computed from the gray matter segment obtained at an early stage of the VBM analysis (before volume modulation). The former approach is more sensitive to local gray matter volume changes, but such changes may not be sufficiently large enough to be reflected in the total gray matter volume, which is simply a sum of all voxels segmented as gray matter. The observed significant decreases of regional gray matter volume in the MF group may also be accompanied by increases in some gray matter regions that did not form statistically meaningful clusters (such as at $P<.001$ uncorrected with a cluster size of $\geq 30$ in Fig $2 B$ or at $P<.05$ corrected in Fig $2 C,-D)$. Therefore, it is possible that in our study, the total gray matter volume was not different between groups but the regional comparison of gray matter showed only significant decreases in the MF group.

Our fMRI results also indicated that BF children may have better functional development of gray matter than MF children. Specifically, when we compared the average activation maps generated at a statistical threshold of $P<.001$ (Bonferroni-corrected) between groups, BF children had significantly more activation in the right frontal and left/right temporal lobes for the perception task and in the left temporal lobe for the language task. Greater brain activation for similar tasks has been observed in healthy adults versus children ${ }^{20}$ and correlated with better task performance, ${ }^{23}$ while reduced activation has been observed in children with dyslexia, ${ }^{24}$ indicating that more activation is probably associated with better functional development of the brain. The overall greater brain activation and presumably better functional development in $\mathrm{BF}$ than in MF children was consistent with the fMRI task performance data in our study, which showed significantly better task performance in BF than in MF children and agreed with literature findings in larger scale studies in which better language development and higher IQ associated with breastfeeding were observed. ${ }^{1,17}$ Greater activation in the left temporal lobe for both tasks in BF children was also consistent with our VBM findings, in which higher regional gray matter volume was detected. The average language fMRI activation maps 
for all children were consistent with activation maps reported for similar visual rhyming tasks for children, ${ }^{20}$ and the BF/MF brain activation differences were significant in the left temporal lobe, which is important for phonologic processing (rhyming in our study) ${ }^{20}$ and involves the fusiform gyrus, which has been regarded as an important region for visual word processing. ${ }^{25}$ These results also suggest that the visual rhyming-language fMRI may be sensitive to differences in language processing associated with infant diets, despite the fact that the language measures such as verbal IQ and CELF-4 scores did not differ significantly (except for a trend toward lower scores in MF children for all language components) in our relatively small cohort of BF and MF children.

One limitation of our study is that we could not obtain family socioeconomic status data for some of the subjects and incorporate the background information into covariate analyses. However, the data we were able to collect of parents' education and income from the subjects were not different between groups ( $\mathrm{Ta}-$ ble 1). Variables not considered in our study during infancy to 8 years of age may also impact brain development in children and may be confounders for our observed association between infant diets and brain development. Nevertheless, the diverse nature of our recruiting methods (advertisements at health fairs, pediatrician offices, summer camps, schools, social media, and community events) might be expected to lessen the confounding influence of these variables in our study. Another study limitation is our relatively small sample size, particularly because we were measuring potentially subtle effects in healthy children. However, we did find statistically significant VBM and fMRI differences between our cohorts of BF and MF children. While we did include sex as a covariate in our analyses, possible cross-sex variations in the effects of infant diets on gray matter development may need to be investigated with a larger sample size. Future studies with larger sample sizes and well-characterized cohorts may be beneficial to better delineate neuroimaging correlates of infant diet effects on children's brain development.

\section{CONCLUSIONS}

We used VBM and fMRI to evaluate the brain gray matter structure and function in healthy 8 -year-old children who were predominantly BF or MF during infancy. Our imaging results showed that BF children had greater gray matter volume in specific brain regions than MF children, despite the total gray matter volume not being different between groups. Our results also showed more brain activation for the BF children when processing perception and language tasks. Our results indicate an association between infant diets and long-term brain development in healthy children.

\section{ACKNOWLEDGMENTS}

We appreciate the Human Studies Core of the Arkansas Children's Nutrition Center for all their effort in this project. We also thank all subjects and their families for participating in this study.

Disclosures: Xiawei Ou—RELATED: Grant: US Department of Agriculture, ${ }^{*}$ Comments: This project is supported by US Department of Agriculture/Agricultural Research Service funding to Arkansas Children's Nutrition Center. Aline AndresRELATED: Grant: US Department of Agriculture/Agricultural Research Service grant.* Rudolph T. Pivik—RELATED: Grant: US Department of Agriculture/Agricul- tural Research Service.* Jeffrey H. Snow—RELATED: Grant: US Department of Agriculture funding of the Nutrition Center.* Thomas M. Badger-RELATED: Grant: US Department of Agriculture, ${ }^{\star}$ Comments: This is a center grant for the Arkansas Children's Nutrition Center to study the effects of diets and nutritional status on child development and disease prevention. *Money paid to the institution.

\section{REFERENCES}

1. Anderson JW, Johnstone BM, Remley DT. Breast-feeding and cognitive development: a meta-analysis. Am J Clin Nutr1999;70:525-35 Medline

2. Brion MJ, Lawlor DA, Matijasevich A, et al. What are the causal effects of breastfeeding on IQ, obesity and blood pressure? Evidence from comparing high-income with middle-income cohorts. Int J Epidemiol 2011;40:670-80 CrossRef Medline

3. Kramer MS, Aboud F, Mironova E, et al; Promotion of Breastfeeding Intervention Trial (PROBIT) Study Group. Breastfeeding and child cognitive development: new evidence from a large randomized trial. Arch Gen Psychiatry 2008;65:578-84 CrossRef Medline

4. Oddy WH, Li JH, Whitehouse AJO, et al. Breastfeeding duration and academic achievement at $\mathbf{1 0}$ years. Pediatrics 2011;127:e137-45 CrossRef Medline

5. Quigley MA, Hockley C, Carson C, et al. Breastfeeding is associated with improved child cognitive development: a population-based cohort study. J Pediatr 2012;160:25-32 CrossRef Medline

6. Isaacs EB, Fischl BR, Quinn BT, et al. Impact of breast milk on intelligence quotient, brain size, and white matter development. Pediatr Res 2010;67:357-62 CrossRef Medline

7. Kafouri S, Kramer M, Leonard G, et al. Breastfeeding and brain structure in adolescence. Int J Epidemiol 2013;42:150-59 CrossRef Medline

8. Deoni SC, Dean DC 3rd, Piryatinsky I, et al. Breastfeeding and early white matter development: a cross-sectional study. Neuroimage 2013;82:77-86 CrossRef Medline

9. Ou X, Andres A, Cleves MA, et al. Sex-specific association between infant diet and white matter integrity in in 8-y-old children. Pediatr Res 2014;76:535-43 CrossRef Medline

10. Isaacs EB. Neuroimaging, a new tool for investigating the effects of early diet on cognitive and brain development. Front Hum Neurosci 2013;7:445 CrossRef Medline

11. Ashburner J, Friston KJ. Voxel-based morphometry: the methods. Neuroimage 2000;11:805-21 CrossRef Medline

12. Adleman NE, Fromm SJ, Razdan V, et al. Cross-sectional and longitudinal abnormalities in brain structure in children with severe mood dysregulation or bipolar disorder. J Child Psychol Psychiatry 2012;53:1149-56 CrossRef Medline

13. Boddaert N, Chabane N, Gervais H, et al. Superior temporal sulcus anatomical abnormalities in childhood autism: a voxelbased morphometry MRI study. Neuroimage 2004;23:364-69 CrossRef Medline

14. Eckert MA, Leonard CM, Wilke M, et al. Anatomical signatures of dyslexia in children: unique information from manual and voxel based morphometry brain measures. Cortex 2005;41:304-15 CrossRef Medline

15. Taki Y, Hashizume H, Sassa Y, et al. Breakfast staple types affect brain gray matter volume and cognitive function in healthy children. PLoS One 2010;5:e15213 CrossRef Medline

16. Dee DL, Li RW, Lee LC, et al. Associations between breastfeeding practices and young children's language and motor skill development. Pediatrics 2007;119:S92-98 CrossRef Medline

17. Belfort MB, Rifas-Shiman SL, Kleinman KP, et al. Infant feeding and childhood cognition at ages 3 and 7 years: effects of breastfeeding duration and exclusivity. JAMA Pediatr 2013;167:836-44 CrossRef Medline

18. Wilke M, Holland SK, Altaye M, et al. Template-O-Matic: a toolbox for creating customized pediatric templates. Neuroimage 2008;41: 903-13 CrossRef Medline

19. Reiss AL, Abrams MT, Singer HS, et al. Brain development, gender and IQ in children: a volumetric imaging study. Brain 1996;119: 1763-74 CrossRef Medline

20. Booth JR, Burman DD, Meyer JR, et al. Development of brain mech- 
anisms for processing orthographic and phonologic representations. J Cogn Neurosci 2004;16:1234-49 CrossRef Medline

21. Herba CM, Roza S, Govaert P, et al. Breastfeeding and early brain development: the Generation R study. Matern Child Nutr 2013;9: 332-49 CrossRef Medline

22. Tzarouchi LC, Astrakas LG, Xydis V, et al. Age-related grey matter changes in preterm infants: an MRI study. Neuroimage 2009;47: 1148-53 CrossRef Medline

23. Booth JR, Burman DD, Meyer JR, et al. Relation between brain acti- vation and lexical performance. Hum Brain Mapp 2003;19:155-69 CrossRef Medline

24. Cao F, Bitan T, Chou TL, et al. Deficient orthographic and phonological representations in children with dyslexia revealed by brain activation patterns. J Child Psychol Psychiatry 2006;47: 1041-50 CrossRef Medline

25. McCandliss BD, Cohen L, Dehaene S. The visual word form area: expertise for reading in the fusiform gyrus. Trends Cogn Sci 2003;7: 293-99 CrossRef Medline 